This is the accepted version of the following article: Guo, Wusheng, et al.

"Rhodium nanoflowers stabilized by a nitrogen-rich PEG-tagged substrate as recyclable catalyst for the stereoselective hydrosilylation of internal alkynes" in Advanced synthesis and catalysis, vol. 357, issue 1, (Jan. 2015), p. 89-99, which has been published in final form at DOI 10.1002/adsc. 201400740. This article may be used for non-commercial purposes in accordance with Wiley Terms and Conditions for Self-Archiving.

DOI: 10.1002/adsc.201((will be filled in by the editorial staff))

\title{
Rhodium nanoflowers stabilized by a nitrogen-rich PEG-tagged substrate as recyclable catalyst for the stereoselective hydrosilylation of internal alkynes
}

\author{
Wusheng Guo, ${ }^{a}$ Roser Pleixats, ${ }^{a *}$ Alexandr Shafir, ${ }^{a, b *}$ and Teodor Parella ${ }^{a, c}$

\begin{abstract}
a Departament de Química and Centro de Innovación en Química Avanzada (ORFEO-CINQA). Universitat Autònoma de Barcelona, Cerdanyola del Vallès, 08193-Barcelona, Spain. Fax: 3493581 2477; Tel: 3493581 2067; E-mail: roser.pleixats@uab.cat; alexandr.shafir@uab.cat Institute of Chemical Research of Catalonia (ICIQ), Av. Països Catalans 16, 43007-Tarragona, Spain.
\end{abstract} \\ Servei de Ressonància Magnètica Nuclear, Universitat Autònoma de Barcelona, Cerdanyola del Vallès, 08193-Barcelona, Spain.
}

Received: ((will be filled in by the editorial staff))

Supporting information for this article is available on the WWW under http://dx.doi.org/10.1002/adsc.201\#\#\#\#\#\#.

\begin{tabular}{ll}
\hline Abstract. Morphology and size controllable rhodium & affording the $(E)$-vinylsilanes in quantitative yields for a \\
nanoparticles stabilized by a nitrogen-rich & wide range of substrates. The insolubility of the \\
polyoxyethylenated derivative have been prepared by nanocatalyst in diethyl ether allows its easy separation and & \\
reduction of $\mathrm{RhCl}_{3}$ with $\mathrm{NaBH}_{4}$ in water at room temperature & recycling. \\
and fully characterized. The flower-like Rh NPs are effective & Keywords: alkynes; hydrosilylation; rhodium \\
and recyclable catalysts for the stereoselective & nanoparticles; polyoxyethylenated compound; recyclable \\
hydrosilylation of challenging internal alkynes and diynes, & catalyst
\end{tabular}

\section{Introduction}

The transition-metal catalyzed hydrosilylation of alkynes represents a highly straightforward, convenient and atom-efficient route for the preparation of vinylsilanes, versatile organosilicon compounds with multiple applications in organic synthesis. ${ }^{[1]}$ Thus, these valuable building blocks are involved in a range of transformations, such as oxidative rearrangement to ketones, vinyl iodide formation or protodesilylation to produce the corresponding alkene. ${ }^{[2]}$ They are also substrates for the Hiyama cross-coupling with vinyl and aryl halides, ${ }^{[3]}$ and for the Tamao-Fleming oxidation to give carbonyl derivatives. ${ }^{[4]}$ In particular, the use of vinylsilanes as nucleophilic vinyl precursors offer advantages over other metal alkenylates in terms of cost, non-toxicity, functional group tolerance and high chemical stability. Certain platinum species, particularly $\mathrm{H}_{2} \mathrm{PtCl}_{6}$ (the Speier's catalyst) and $\mathrm{Pt}_{2}\left[\left(\mathrm{Me}_{2} \mathrm{SiCH}=\mathrm{CH}_{2}\right)_{2} \mathrm{O}\right]_{3}$ (the Karstedt's catalyst) have been the traditional catalyst choice for the addition of silanes to unsaturated C-C bonds. ${ }^{[5]}$ More recently, ruthenium complexes have been introduced as alternatives to the Pt-based systems. ${ }^{[6]}$ Although terminal acetylenes tend to show higher reactivity with both catalyst families, few catalytic systems (i.e. Trost's ruthenium catalyst) ${ }^{[\mathrm{gg}]}$ perform well with the more challenging internal alkynes. In this context, although rhodium-catalyzed hydrosilylation remains relatively unexplored, a handful of reports have appeared describing the reaction of both the terminal and internal alkynes with silanes under catalysis by $\mathrm{Rh}(\mathrm{I})$ complexes, ${ }^{[7]}$ including $[\mathrm{Rh}(\mathrm{COD}) \mathrm{Cl}]_{2}$, $[\mathrm{RhCl}(\mathrm{nbd})]_{2} / \mathrm{dppp}$ and $\left.\mathrm{RhCl}\left(\mathrm{PPh}_{3}\right)_{3}\right)$; in addition, Rh-NHC species have been used for the hydrosilylation of ketones ${ }^{[8 a]}$ and terminal alkynes, ${ }^{[8 b, c]}$ and molecular $\mathrm{Rh}(0)$ complexes confined within carbon nanoreactors have been evaluated in the hydrosilylation of phenylacetylene. ${ }^{[8 \mathrm{~d}]}$

In addition to discrete complexes, a few recent examples have appeared dealing with the use of metal nanoparticles in the addition of silanes to acetylenic compounds. ${ }^{[9]}$ Thus, Yus et al described the recyclability of platinum nanoparticles deposited on titania ${ }^{[9 a, b]}$ and on magnetite ${ }^{[9 c]}$ in the hydrosilylation of internal alkynes and diynes. More recently, Pt nanoparticles in carbon nanoreactors have been used in the hydrosilylation of 
phenylacetylene, allowing for the evaluation of the effect of nanoscale confinement on the process selectivity.

${ }^{[9 d]}$ In 2014 we have reported ${ }^{[9]}$ the hydrosilylation of a range of internal alkynes catalyzed by Pd nanoparticles stabilized by tris-imidazolium salts (Figure 1, stabilizer A) which affords the syn-addition products in a stereoselective manner. As a particular feature, however, the system was found highly sensitive to moisture, and rigorously anhydrous conditions were required to obtain the vinylsilanes. In the presence of water a competitive transfer hydrogenation took place to give the corresponding alkenes and/or alkanes. ${ }^{[9]}$

In addition, supported rhodium, ${ }^{9 f}$ rhodium-platinum ${ }^{[9 f]}$ and gold nanoparticles ${ }^{[9 g, h]}$ have been described as catalysts for the regioselective hydrosilylation of alkynes. During that research we were striken by the scarcity of reports ${ }^{[9, i]}$ on catalytic rhodium nanoparticles (Rh NPs) for the hydrosilylation of internal alkynes, given that the use of such materials as catalysts has been quickly expanding to a great deal of other transformations. ${ }^{[10]}$

Our promising results on the activity of the tris-imidazolium stabilized Pd nanoparticles (Figure 1 A) in alkyne hydrosilylation ${ }^{[9 e]}$ encouraged us to pursue the research on other metal nanoparticles as catalysts for this transformation. As part of this effort, a new nanoparticle stabilizer was developed featuring three PEG chains bound to a central three-fold symmetric core, with the hopes that the poly-ether chains would help with the catalyst recycling. ${ }^{[11]}$ The few existing reports on Rh complexes ${ }^{[7,8]}$ and the scarce precedents ${ }^{[9]]}$ on the use of Rh NPs in hydrosilylation led us to design a PEG-tagged derivative suitable for the development of a recyclable alkyne hydrosilylation catalyst based on Rh NPs.

Figure 1. Stabilizers for metal nanoparticles.

Applying our group’s previous experience ${ }^{[11 c]}$ whereby a PEG-matrix prepared via the copper-catalysed alkyne-azide cycloaddition (CuAAC) chemistry (Figure $1 \mathrm{~B}$ ) was used as a stabilizer in the synthesis of water soluble but diethyl ether insoluble Pd NPs, here a nitrogen-rich polyoxyethylenated derivative 1 (Figure 1 C) was envisaged for the formation of Rh NPs. This new three-fold molecular architecture was proposed taking into account both the potential benefits of the nitrogen-enriched structure on the nanoparticle stabilization, and the operational convenience of bonding via the the CuAAC click linkages. ${ }^{[12]}$

We describe herein the synthesis of the new material $\mathbf{1}$ and its use in the efficient preparation of size and morphology controllable Rh NPs. The catalytic activity and recyclability of the Rh NPs thus obtained in the hydrosilylation of various alkynes is also described. To the best of our knowledge, this is the first report on the use of the Rh NPs in the hydrosilylation of the challenging internal alkynes.

\section{Results and Discussion}

The preparation of stabilizer $\mathbf{1}$ and the corresponding Rh NPs are summarized in Scheme 1. Using a modification of the group's earlier methodology, ${ }^{[11 c]}$ the commercially available polyether MeO-PEG-OH was treated with methanesulfonyl chloride in the presence of triethylamine to give the corresponding mesylate $\mathbf{2}$ in $94 \%$ yield. This was then allowed to react with sodium azide in dimethylformamide at $60{ }^{\circ} \mathrm{C}$ overnight, affording the azide 3 in a quantitative yield. The second coupling partner, 2,4,6-tris(propargylamino)-1,3,5triazine, $\mathbf{5}$, was easily obtained in one step from either cyanuric chloride $\mathbf{4 a}$ or cyanuric fluoride $\mathbf{4 b}$ with $34 \%$ and $83 \%$ yields, respectively. ${ }^{[13]}$ Despite the better performance, caution should be taken when using the cyanuric fluoride due to its highly unpleasant smell. With the trialkyne $\mathbf{5}$ and the azide $\mathbf{3}$ in hand, we went on to perform a threefold copper-catalyzed 1,3-dipolar cycloaddition reaction using a 1:3 molar ratio. After testing several catalytic system, ${ }^{[14]}$ best results were achieved using a method developed by Cattoën et al. using $\mathrm{CuBr}\left(\mathrm{PPh}_{3}\right)$ as catalyst under microwave irradiation. ${ }^{[15]}$ The reaction progress was monitored by IR, with completion of the reaction signaled by the disappearance of the azide absorption around $2099 \mathrm{~cm}^{-1}$. 
Scheme 1. Preparation of Rh NPs stabilized by 1.

A chemical reduction method was then applied to test the stabilizing ability of $\mathbf{1}$ for the formation of Rh NPs using $\mathrm{RhCl}_{3} \cdot 3 \mathrm{H}_{2} \mathrm{O}$ as the metal source and $\mathrm{NaBH}_{4}$ as a reducing reagent, in water at room temperature (Table 1).

Initially, the nanoparticle formation was tested using a 1:1 $\mathbf{1} / \mathrm{Rh}$ ratio (Table 1 , entry 1 ). The addition of $\mathrm{NaBH}_{4}$ to a well stirred solution of $\mathbf{1}$ and $\mathrm{RhCl}_{3} \cdot 3 \mathrm{H}_{2} \mathrm{O}$ immediately led to a color change from light brown to black, but without any precipitate of bulk rhodium appearing after stirring overnight. TEM analysis of the resulting mixture showed the formation of the Rh NPs with a mean diameter of $1.6 \mathrm{~nm}$. To probe the influence of the stabilizer-to-metal ratio on the nanoparticle morphology and reactivity, additional syntheses were conducted varying the ratio $\mathbf{1} / \mathrm{Rh}$ from $0.3: 1$ to $0.02: 1$ (Table 1 , entries 2-5). In all cases, the corresponding nanoparticles were obtained in good yields with respect to the rhodium precursor, and with \%Rh (by ICP) comparable to the theoretical percentage. As a reference, the reduction of $\mathrm{RhCl}_{3} \cdot 3 \mathrm{H}_{2} \mathrm{O}$ with $\mathrm{NaBH}_{4}$ in the absence of 1 gave bulk rhodium precipitate (Table1, entry 6).

Table 1. Preparation of Rh NPs stabilized with different amounts of stabilizer $\mathbf{1}$.

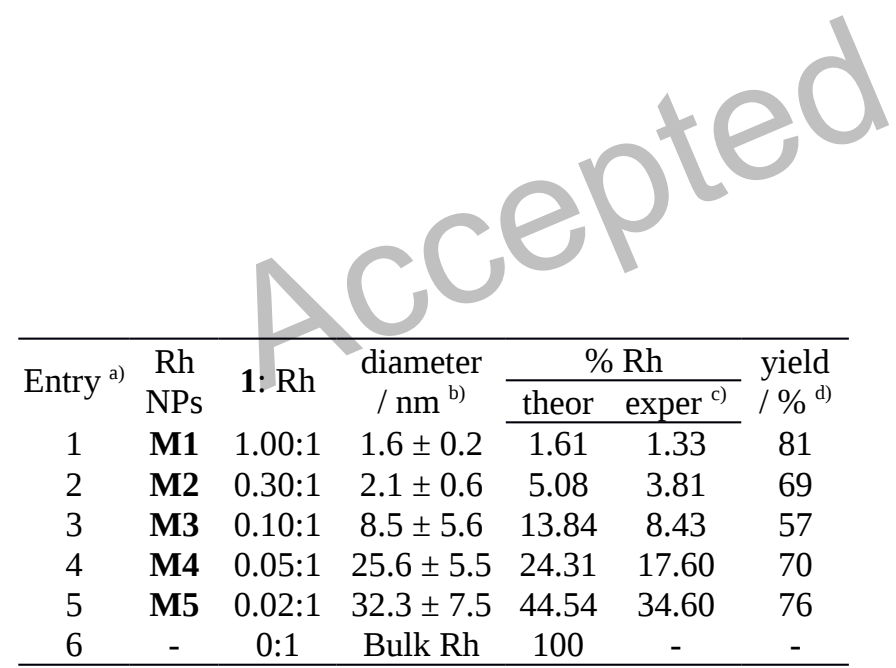

a) Reaction conditions: [Rh] = $1 \mathrm{mM} ; \mathrm{RhCl}_{3}$ and $\mathbf{1}$ stirred in water overnight under $\mathrm{N}_{2}$; ${ }^{\text {b) }}$ see supporting information for mean diameter (by TEM) and size distributions; ${ }^{c}$ determined by ICP; ${ }^{\text {d) }}$ based on $\mathrm{RhCl}_{3}$ used. 


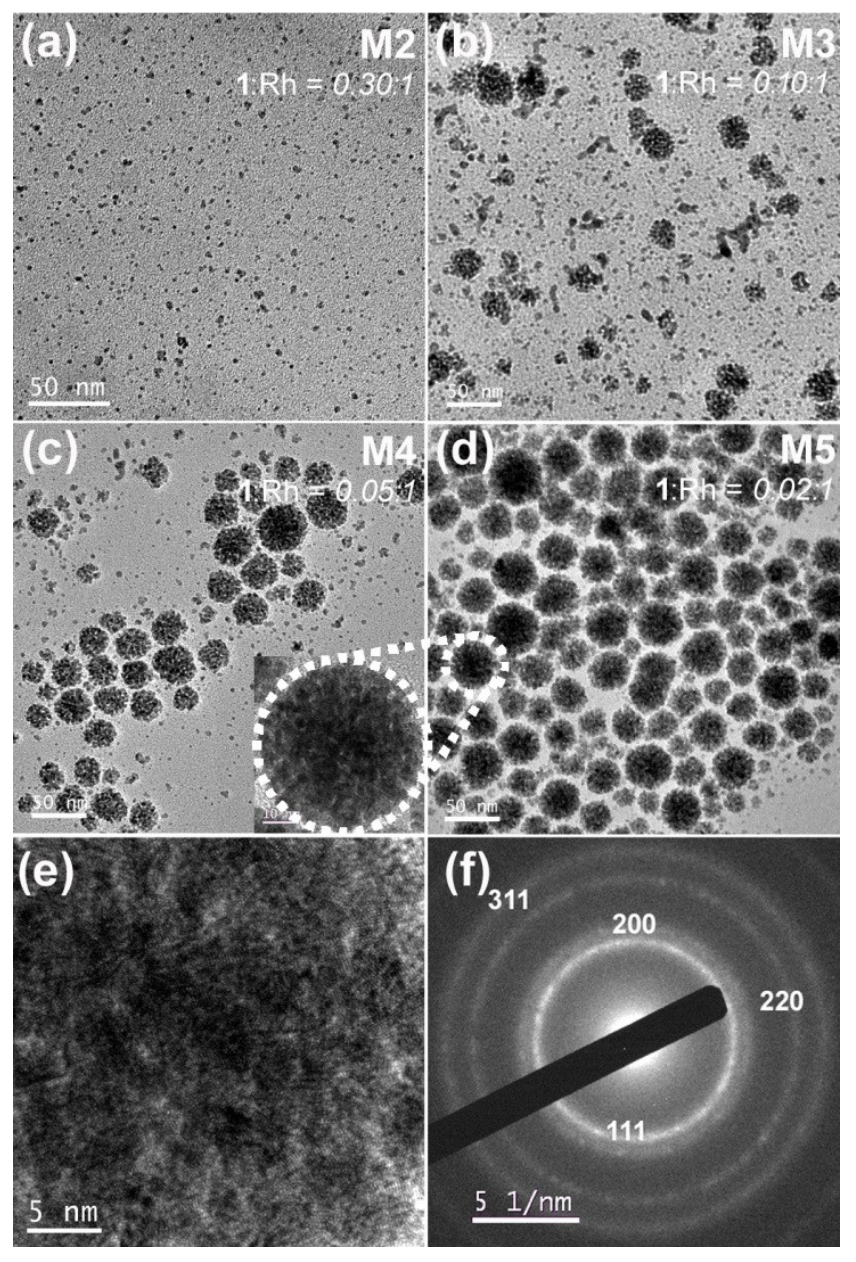

Figure 2. (a-d) Correspond to TEM images of M2, M3, M4 and M5, respectively; M1 has similar morphology as M2, but with smaller average particle size; (e) HRTEM image of the "nanoflower" encircled in (d); (f) SAED pattern of the same "nanoflower" in (d).

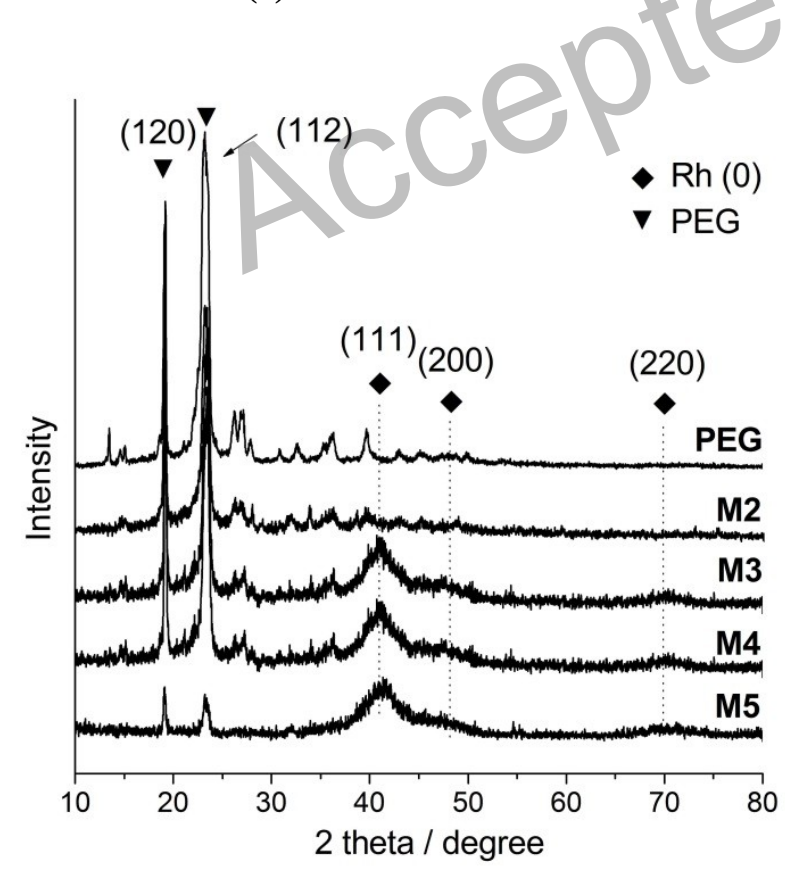

Figure 3. $p$-XRD patterns of PEG-2000, M2, M3, M4 and M5.

Notably, upon decreasing the stabilizer-to-metal ratio, the particles underwent a diameter increase from 1.6 to $32.3 \mathrm{~nm}$ and a concomitant morphology change from small clusters to flower-like nanostructures (Figure 2 ad). ${ }^{[16]}$ Specifically, figures $2 e$ and $2 f$ contain the high resolution TEM (HRTEM) image and the selected area electron diffraction (SAED) pattern of the "nanoflower" structure outlined in Figure 2d, showing the face- 
centered cubic (fcc) $\mathrm{Rh}(0)$ lattice in M5. The powder X-ray diffraction ( $p$-XRD) patterns of M2, M3, M4 and M5, along with that for the commercial PEG-2000 reference, are shown in Figure 3. Here, the broad peaks at around $2 \theta=41,48$ and $70^{\circ}$ correspond to the fcc rhodium(0) planes (111), (200) and (220), respectively, ${ }^{[17 a]}$ and the two sharper peaks at $2 \theta=19$ and $23^{\circ}$ are ascribed to the (120) and (112) planes of PEG-chains. ${ }^{[17 b]}$ The absence of discernible $\mathrm{Rh}(0)$ signals in $\mathbf{M} 2$ is due to its low rhodium content.

With the Rh NPs M1-M5 in hand, the hydrosilylation of diphenylacetylene with $\mathrm{Et}_{3} \mathrm{SiH}$ was chosen as the model reaction under a range of conditions (Table 2). In all cases, the neat silane (4 equiv) was used as the reaction medium, a feature we found beneficial and convenient in Pd-catalyzed hydrosilylation. ${ }^{[9]}$ Thus, a mixture of diphenylacetylene and triethylsilane was treated with $\mathbf{M 5}(0.5 \mathrm{~mol} \% \mathrm{Rh}$ loading $)$ at $90{ }^{\circ} \mathrm{C}$ under inert atmosphere for $17 \mathrm{~h}$. Under these conditions, the alkyne was fully consumed (as gauged by GC), with the corresponding syn addition product (E)-6a obtained in quantitative yield (Table 2, entry 1).

The quantitative formation of (E)-6a was maintained upon lowering the temperature to $60{ }^{\circ} \mathrm{C}$ (Table 2, entry 2); however, further decreasing the temperature to $40{ }^{\circ} \mathrm{C}$ led to a poor conversion after $20 \mathrm{~h}$ (Table 2, entry 3). Notably, lowering the catalyst loading from $0.5 \mathrm{~mol} \%$ to just $0.1 \mathrm{~mol} \% \mathrm{Rh}$ at $60{ }^{\circ} \mathrm{C}$

Table 2. Optimization of the reaction conditions for the hydrosilylation of diphenylacetylene with triethylsilane under Rh NPs catalysis.

\begin{tabular}{|c|c|c|c|c|c|c|}
\hline Entry a) & catalyst & $\begin{array}{c}\mathrm{Rh} \\
\mathrm{mol} \% \\
\end{array}$ & $\begin{array}{c}\text { solvent } \\
\text { / mL }\end{array}$ & $\begin{array}{c}\mathrm{T} \\
/{ }^{\circ} \mathrm{C} \\
\end{array}$ & $\begin{array}{c}\text { conv. } \\
\end{array}$ & $\begin{array}{l}\text { yield } \\
1 \%^{\mathrm{b})} \\
\end{array}$ \\
\hline $1^{\text {c) }}$ & M5 & 0.5 & - & 90 & $>99$ & 99 \\
\hline 2 & M5 & 0.5 & - & 60 & $>99$ & 99 \\
\hline 3 & M5 & 0.5 & - & 40 & $19^{\mathrm{d})}$ & $17^{d}$ \\
\hline $4^{e)}$ & M5 & 0.1 & - & 60 & & 99 \\
\hline 5 & M5 & 0.5 & ${ }_{2} \mathrm{O} / 0.036$ & 60 & $>99$ & 99 \\
\hline $6^{f)}$ & M5 & 0.5 & - & 60 & $>99$ & 99 \\
\hline & M5 & 0.5 & EtOH / 1 & 60 & $>99$ & 99 \\
\hline 8 & M5 & 0.5 & $\mathrm{CHCl}_{3} / 1$ & 60 & $>99$ & 99 \\
\hline 9 & M4 & 0.5 & - & 60 & $>99$ & 99 \\
\hline 10 & M3 & 0.5 & - & 60 & 0 & - \\
\hline 11 & M2 & 0.5 & - & 60 & 0 & - \\
\hline 12 & M1 & 0.5 & - & 60 & 0 & - \\
\hline 13 & - & - & - & 60 & 0 & - \\
\hline
\end{tabular}

a) Performed in closed vessels $(15 \mathrm{~mL})$ with $0.5 \mathrm{mmol}$ of alkyne and $\mathrm{HSiEt}_{3}(0.320 \mathrm{~mL}, 2 \mathrm{mmol}) ;{ }^{\text {b) }}$ isolated yield; ${ }^{\mathrm{c}}$ experiments of entries 1-5 were performed under dry $\mathrm{N}_{2}$; ${ }^{\text {d) }}$ determined by GC with undecane as internal standard; ${ }^{\text {e) }}$ performed with $6.0 \mathrm{mmol}$ of alkyne; ${ }^{\mathrm{f}}$ experiments of entries 6-13 were performed under air.

still allowed for a fully selective conversion (Table 2, entry 4), with the catalyst reaching a respectable turnover number (TON $=990)$. In the latter case the reaction was conducted on a $6 \mathrm{mmol}$ scale. A trial was also conducted in the presence of added water (Table 2, entry 5, using $2 \mathrm{mmol}, 4$ eq. of $\mathrm{H}_{2} \mathrm{O}$ ), in order to determine whether the new rhodium catalyst would then switch to a hydrogenation mode, as was the case for the Pd NPs. ${ }^{[9]}$ Interestingly, unlike with the Pd system, no corresponding hydrogenated product was detected, and the alkyne was once again cleanly converted to vinylsilane $(E)-6 a$, showcasing the catalyst's water tolerance. In fact, inert atmosphere was shown to be unnecessary (Table 2, entry 6), and the system was not affected by the addition of solvents, e. g., ethanol or chloroform (Table 2, entries 7-8). Good activity was also observed for the 
Rh NPs corresponding to M4 (Table 2, entry 9); however, no conversion was observed using M3, M2 or M1 (Table 2, entries 10-12), likely due to the higher 1:Rh ratio in these materials, as shown previously by others. ${ }^{[18]}$ A control experiment showed that no reaction took place in the absence of a catalyst (Table 2, entry 13).

Having established Rh NPs M4 and M5 as the best catalysts, M5 was chosen to achieve the hydrosilylation of a variety of symmetrical internal alkynes under the optimized conditions ( $0.5 \mathrm{~mol} \% \mathrm{Rh}, 4$ eq. of silane, 60 $\left.{ }^{\circ} \mathrm{C}\right),{ }^{[19]}$ as shown in Table 3 . The protocol proved to be highly efficient and selective, furnishing the corresponding $(E)$-alkenylsilanes $(E)$-6 in excellent yields for substrates bearing electron-withdrawing or electron-donating substituents on the aromatic ring (Table 3, entries 1-7), as well as with the non-aromatic substrates (Table 3, entries 8-9).

Table 3. Hydrosilylation of symmetric alkynes under catalysis by M5. ${ }^{a}$ 